Revue internationale P.M.E.

Économie et gestion de la petite et moyenne entreprise

\title{
Dans quelles mesures les TIC jouent-elles un rôle stratégique pour les PME ?
}

\section{Béatrice Vacher}

Volume 15, numéro 3-4, 2002

URI : https://id.erudit.org/iderudit/1008812ar

DOI : https://doi.org/10.7202/1008812ar

Aller au sommaire du numéro

\section{Éditeur(s)}

Presses de l’Université du Québec

ISSN

0776-5436 (imprimé)

1918-9699 (numérique)

Découvrir la revue

Citer cet article

Vacher, B. (2002). Dans quelles mesures les TIC jouent-elles un rôle stratégique pour les PME ? Revue internationale P.M.E., 15(3-4), 37-61.

https://doi.org/10.7202/1008812ar
Résumé de l'article

Dans cet article, nous précisons le lien entre TIC, stratégie et organisation du travail dans les PME à travers l'étude des fonctions des TIC. L'objectif est de mieux comprendre les comportements des PME en matière d'utilisation des TIC et d'en anticiper les évolutions.

Nous utilisons une grille de lecture montrant que l'information a non seulement des fonctions opérationnelles (fabriquer ou vendre mieux, moins cher, etc.), mais également sociales (humaines, organisationnelles). Elle sert aussi à montrer ou afficher une certaine efficacité ou qualité et à rassurer ou se rassurer. À partir de cette grille, nous posons la question de savoir à quoi servent les TIC pour les PME et nous dégageons cinq comportements types plus ou moins cohérents avec la stratégie d'entreprise et à partir desquels des préconisations d'action peuvent être faites : 1) les « intégrées ", qui ont une vision à long terme cohérente entre l'usage des TIC et leur stratégie, 2) les "sous pression ", poussées par l'environnement et peu sensibles aux questions sociales, 3) les « borgnes », pour qui les TIC sont plus encombrantes qu'utiles, au moins pour une activité, 4) les « fébriles " et 5) les « hyperactives ", qui on besoin de clarifier leur positionnement stratégique.
Ce document est protégé par la loi sur le droit d'auteur. L'utilisation des services d'Érudit (y compris la reproduction) est assujettie à sa politique d'utilisation que vous pouvez consulter en ligne.

https://apropos.erudit.org/fr/usagers/politique-dutilisation/ 


\title{
Dans quelles mesures les TIC jouent-elles un rôle stratégique pour les PME?
}

\author{
Béatrice VACHER \\ École des mines d'Albi-Carmaux
}

\section{MOTS CLÉS}

\section{Technologie de l'information et de la communication (TIC) Petites et moyennes entreprises (PME) - Stratégie d'entreprise Organisation du travail - Fonctions des TIC - Utilisation des TIC}

\section{RÉSUMÉ}

Dans cet article, nous précisons le lien entre TIC, stratégie et organisation du travail dans les PME à travers l'étude des fonctions des TIC. L'objectif est de mieux comprendre les comportements des PME en matière d'utilisation des TIC et d'en anticiper les évolutions.

Nous utilisons une grille de lecture montrant que l'information a non seulement des fonctions opérationnelles (fabriquer ou vendre mieux, moins cher, etc.), mais également sociales (humaines, organisationnelles). Elle sert aussi à montrer ou afficher une certaine efficacité ou qualité et à rassurer ou se rassurer. À partir de cette grille, nous posons la question de savoir à quoi servent les TIC pour les PME et nous dégageons cinq comportements types plus ou moins cohérents avec la stratégie d'entreprise et à partir desquels des préconisations d'action peuvent être faites: 1) les « intégrées », qui ont une vision à long terme cohérente entre l'usage des TIC et leur stratégie, 2) les " sous pression », poussées par l'environnement et

\section{L'AUTEURE}

BÉATRICE VACHER est enseignante-chercheure en gestion et en sciences de l'information et de la communication. Elle travaille depuis dix ans sur l'utilisation, l'organisation et les malentendus de l'information et de ses systèmes et technologies. Jusqu'à présent, elle a adopté une approche qualitative des problématiques (recherche-action en entreprise ou enquêtes de terrain). Elle est rattachée au centre de recherche LGI2P de l'École des mines d'Alès. Adresse : École des mines d'Alès, Site EERIE, Parc scientifique Georges-Besse, F 30035 Nîmes Cedex 1. Enfin, elle est chercheure associée au LERASS de l'Université Toulouse III Paul-Sabatier. Courriel : beatrice.vacher@ema.fr 
peu sensibles aux questions sociales, 3) les «borgnes », pour qui les TIC sont plus encombrantes qu'utiles, au moins pour une activité, 4) les «fébriles "et 5) les « hyperactives », qui ont besoin de clarifier leur positionnement stratégique.

\begin{abstract}
In this article, we point out the connection between CIT (Communication and Information Technologies), strategy and labour organization specific to SMEs through the study of the function of CIT. The aim is to better understand the behaviour of SME in dealing with the use of CIT and to anticipate their developments.

We use an interpretation grid presenting four functions of information, its management and technologies : not only are the functions of information operational (to make or sell better, cheaper and so on) but also social. Information is also useful to show a certain efficiency or quality and to reassure or reassure oneself.

1) The «integrated» have a coherent long term vision between the use of CIT and their own strategy, 2) the "under-pressures", driven by the environment and not very sensitive to social issues, 3) the "one-eyed", for whom CIT is more embarrassing than useful at least for one activity, 4) the "feverish" and 5) the "hyperactive", who need to clarify their strategic position.

From this perspective, we show the functions of CIT for SMEs and define five typical behavioural patterns which are more or less coherent with the strategy of the company : some recommendations for steps to be taken can be infered.
\end{abstract}

\title{
RESUMEN
}

En este articulo, precisamos la conexion entre las TIC, la estrategia y la organizacion del trabajo especificas de la PME a través del estudio de las funciones de las TIC. El objectivo es comprender mejor los comportamientos de las PME en lo tocante a la utilizacion de las TIC y anticipar las evoluciones de éstas.

Utilizamos una tabla de lectura que presenta cuatro funciones de la informacion: la informacion no tiene solamente funciones operativas (fabricar o vender mejor, mas barato, etc.), sino tambien, sociales. Sirve también para mostrar o anunciar cierta eficacia o calidad y a tranquilizar o tranquilizarse. A partir de esta tabla, nos preguntamos para qué sirven las TIC en las PME y extraemos cinco comportamientos mas o menos coherentes con la estrategia de empresa y a partir de los cuales pueden hacerce algunas recomendaciones: 1) los «integrados", que tienen una vision, a largo plazo, coherente entre la utilizacion de las TIC y su estrategia, 2) los «bajo presion», favorecidos por el entorno y poco sensibles a las cuestiones sociales, 3) los «tuertos " para los cuales las TIC son mas molestas que utiles, al menos para una actividad, 4) los «febriles » y 5) los « hyperactivos » que necesitan clarificar su posicion estratégica.

\section{ZUSAMMENFASSUNG}

In diesem Artikel klären wir den Zusammenhang auf zwischen den Informationsund Kommunikationstechnologien, der Strategie und der Arbeitsorganisation in den KMU quer durch die Studien über Funktionen von Informations- und Kommuni- 
kationstechnologien. Ziel ist es, die Verhaltensweisen der KMU in der Anwendung von Informations- und Kommunikationstechnologien besser zu verstehen und die Entwicklungen vorherzusehen. Wir betrachten die Informationen nicht nur aus einer operationellen (besser produzieren oder besser verkaufen, günstiger, etc.) sondern gleichzeitig aus einer sozialen Sichtweise (Menschen, Organisationen). Diese Sichtweisen erlauben, auch eine gewisse Wirksamkeit oder Qualität der Informations- und Kommunikationstechnologien aufzuzeigen und zu belegen.

Ausgehend von dieser Betrachtung stellen wir die Wissensfrage, welches ist der Zweck der Informations- und Kommunikationstechnologien bei den KMU. Wir legen fünf Verhaltenstypen frei, die mehr oder weniger verbunden sind mit der Strategie der Unternehmen und die erlauben, Aktionsempfehlungen zu machen: 1) die 'Integrierten', die eine langfristig, verbundene Vision haben bei der Anwendung der Informations und Kommunikationstechnologien und ihrer Strategie, 2) die 'Unter-Druck-Gesetzten', getrieben durch die Umwelt und wenig sensibel sind gegenüber sozialen Fragen, 3) die 'Finsteren', für welche die Informationsund Kommunikationstechnologien mehr behindernd als nützlich sind, 4) die 'Fieberhaften', 5) die, 'Hyperaktiven', die eine Klärung ihrer strategischen Positionierung benötigen.

\section{Introduction}

La question du titre s'inscrit dans un courant de recherche qui considère les TIC non pas comme un simple outil mais comme un élément central dans les organisations (Venkatraman, 1995 ; Porter, 2001 ; Jacob, 1999; Brousseau et Rallet, 1997).

Cet article y répond par une approche fonctionnelle qui se veut complémentaire d'autres approches comme celles de Bergeron, Raymond et Rivard (2001) et Bergeron et al. (1998) qui montrent le lien entre la performance des PME et une mise en œuvre stratégique des TI en tenant compte des orientations générales de la PME et de sa structure (les incertitudes de l'environnement jouant un rôle négligeable). Blili et Raymond (1993) préconisaient déjà une gestion stratégique des TI pour les PME par la mobilisation des facteurs clés de succès dans les « cinq forces » de Porter $\left(1999^{1}\right)$. Ouellet (2001) présente une étude très détaillée sur l'usage des TIC par les PME au Québec et conclut sur l'importance pour les PME de développer leur capacité organisationnelle pour intégrer au mieux les TIC ainsi que sur l'art de mobiliser le réseau d'action (associations, centres d'affaires, syndicats, etc.). L'auteur précise qu'un discours et une aide indifférenciés sur les TIC pour les PME est tout à fait inutile, que ces entreprises ont des besoins spécifiques, plus axés sur les questions organisationnelles que techniques. Gadille et d'Iribarne (2000) ainsi qu'Amabile, Gadille et Meissonier (2000) montrent, quant à eux, le

1. La première édition datant de 1985.

Revue internationale P.M.E., vol. 15, nos 3-4, 2002

(C) 2003 - Presses de l'Université du Québec

Édifice Le Delta I, 2875, boul. Laurier, bureau 450, Sainte-Foy, Québec G1V 2M2 - Tél. : (418) 657-4399 - www.puq.uquebec.ca

Tiré de : Revue internationale P.M.E., vol. 15, nos $3-4$, sous la direction de Pierre-André Julien. 
rôle joué par les TIC dans l'agilité et la réactivité des PME face à un environnement versatile. Les TIC accompagnent la gestion au quotidien et l'adaptation des options stratégiques sans être pour autant déclencheurs de changements stratégiques.

Dans tous ces cas, les spécificités des PME sont prises en compte, en particulier l'aspect souvent intuitif de la stratégie, le peu de différenciation entre les services, une grande centralisation (la PME étant souvent l'extension de la personnalité du dirigeant), des réactions rapides avec une gestion de l'information plutôt informelle malgré un environnement financier incertain, l'importance de la proximité, etc. (Julien, 1990, 1994 ; Julien et Marchesnay, 1988 ; Carrière, 1990 ; Girard, 1997 ; Raymond, 2000 ; Julien et al., 1997 ; Torrès, 2000 ; Boutary, 2001 ; Raymond et Blili, 1992).

Notre article se démarque des précédentes approches par la manière de formuler la question: les TIC servent-elles à la PME de façon purement opérationnelle ou trouve-t-on d'autres fonctions tout aussi importantes mais moins palpables ? Et dans quelles mesures ces fonctions sont-elles liées à la stratégie de l'entreprise ?

Notre démarche est inductive et qualitative : nous avons rencontré une quarantaine de personnes dans $20 \mathrm{PME}^{2}$. Nous sommes partis d'un questionnaire classique sur les usages des TIC et nous avons dégagé des discours connexes sur des préoccupations d'ordre organisationnel ou stratégique. Nous avons également repéré des usages a priori très peu opérationnels et que nous avions du mal à analyser par une approche classique : que signifie, par exemple, ce besoin d'utiliser les TIC pour pister ses collaborateurs autant que pour améliorer la relation clientèle? Est-ce compatible? Comment différencier les PME mobilisant les TIC pour amplifier les échanges internes des PME qui en font un usage exclusivement marketing?

Pour répondre à ces questions, nous avons utilisé une grille existante sur les fonctions de l'information et de sa gestion dans les entreprises. Cette grille, présentée dans la section modèle et hypothèse de cet article, permet de classer les PME pour en comprendre les comportements à l'égard des TIC et, en particulier, en relation avec la stratégie et l'organisation. La stratégie telle que nous l'entendons n'est pas forcément explicite comme le recommande par exemple l'école du positionnement (Porter, 1999; Strategor, 1997), mais peut être intuitive (ce qui caractérise effectivement les PME dont celles que nous avons étudiées) en se manifestant notamment par un long discours sur la fierté d'un dirigeant pour son produit ou pour la qualité de ses relations avec les clients. Au même titre que l'information et les TIC ne sont pas toujours directement opérationnelles, la stratégie n'a pas toujours de sens pour l'action directe. Adopter une stratégie et avoir

2. Notre travail fait partie d'une enquête menée avec cinq autres chercheures dont les résultats ont fait l'objet d'un rapport (Boutary et al., 2001 ; Boutary et al., 2002).

Revue internationale P.M.E., vol. 15, ns 3-4, 2002 
un discours correspondant permet aussi de rassembler, se mettre d'accord ou, au contraire, affirmer une position, prendre du pouvoir (Mintzberg, 1989; Morgan, 1996).

Nous considérons également que l'organisation est une composante de la stratégie : c'est l'axe «comment» du triaxe d'Abell (1980) qui complète les axes marketing (le couple client-produit). Nous détaillons dans la section méthodologie les critères qui nous permettent d'étudier ces aspects, en particulier la dimension structurelle, les mécanismes de coordination (dont les outils de gestion), la dimension sociale et le poids de l'information dans l'organisation (Aoki, 1988; Nonaka et Takeuchi, 1995).

Initialement, la grille ne tient pas compte des spécificités des PME. C'est dans la section résultats que nous comprenons pourquoi les PME se situent principalement dans certaines catégories (par rapport notamment aux bureaucraties). En conclusion, nous complétons notre grille en indiquant dans quelles mesures les PME peuvent intégrer au mieux les TIC dans leur stratégie. Nous atteignons là les limites de notre démarche. En effet, nous ne pouvons pas faire des préconisations générales : c'est à partir des discours et observations faites que nous avons mis l'accent sur telle ou telle action qui pourrait être développée. Nous avons simplement fait évoluer notre grille initiale en regroupant ces actions ou efforts à développer sous des dénominations communes afin de prendre du recul par rapport aux résultats directs de ce travail.

\section{Modèle et hypothèse}

La grille de lecture que nous mobilisons a été élaborée à partir de cas de gestion de l'information pour répondre à la question suivante : «À quoi sert l'information dans les entreprises ?» (Vacher, 1997, 2000).

Cette grille montre qu'au-delà des fonctions opérationnelles («fabriquer», c'est-à-dire produire, améliorer la productivité ou la qualité, vendre, vendre plus cher, à meilleur coût, etc.), l'information et sa gestion servent aussi à «montrer » (que l'on fabrique avec qualité, que l'on sait faire), à « socialiser » ou à se socialiser (connaître les autres et se faire connaître, apprendre, aider, donner du sens à ce que l'on fait, etc.) et à «rassurer » ou à se rassurer (garder une information pour asseoir une position, informatiser pour régler des problèmes de communication, accumuler des documents sans même les ouvrir, pouvoir présenter des dossiers censés avoir servi à prendre une décision, etc. $)^{3}$. Les fonctions sont synthétisées dans le tableau suivant.

3. Pour cette dernière fonction, voir Feldman et March (1991) qui décrivent comment l'information est d'abord « un signal et un symbole » (de légitimité, de reconnaissance, etc.).

Revue internationale P.M.E., vol. 15, nos 3-4, 2002 
TABLEAU 1

Les fonctions de l'information, de ses technologies et de sa gestion collective

\begin{tabular}{lcc}
\hline Visibilité & ACTION & PREUVE \\
\hline MANIFESTE & & Montrer \\
LATENTE & $\begin{array}{c}\text { Fabriquer } \\
\text { (Détruire) } \\
\text { Socialiser } \\
\text { (Isoler) }\end{array}$ & $\begin{array}{c}\text { Rassurer } \\
\text { (Perturber) }\end{array}$ \\
\hline
\end{tabular}

Il ne s'agit donc pas de promouvoir une approche fonctionnaliste qui réduirait l'esprit humain à une machine ${ }^{4}$ mais d'adopter une approche complémentaire de celle des usages (Brousseau et Rallet, 1997), de l'action située et de l'interactionnisme ${ }^{5}$, en dégageant, à travers les discours des acteurs de PME, le poids relatif des différentes fonctions, manifestes et latentes (Merton, 1965) des TIC. Ces fonctions dépendent des enjeux plus ou moins partagés des différents acteurs. Ces enjeux sont le plus souvent liés à la stratégie de l'entreprise (même intuitive dans le cas de nombreuses PME), à son contexte socioéconomique, au poids de son histoire et de la culture, à ses ressources, c'est-à-dire à sa structure et ses modes de gestion, à l'implication de son personnel et aux efforts technologiques possibles.

Cette grille a déjà été utilisée par des chefs de projet informatique et des chefs d'entreprise pour comprendre pourquoi et comment de grosses dépenses étaient faites en matière de système d'information «inutile» (au sens des fonctions opérationnelles). Elle montre les liens entre ce qui est manifeste, évident et ce qui est vital sans pour autant être précisé entre une logique de l'action et une logique de la preuve. En effet, les quatre fonctions ne sont pas indépendantes et agissent les unes sur les autres : fabriquer ${ }^{6}$ des produits de qualité sans le montrer est dommage, les concurrents pourraient prouver qu'ils font de la qualité sans pour autant en faire (Mispelblom-Beyer, 1999; Vacher, 2001). Peut-on fabriquer longtemps sans rassurer et socialiser? C'est-à-dire sans créer de la confiance dans son personnel ou dans sa hiérarchie, sans être sûr des outils que l'on mobilise ou en ayant peur des conséquences de chacune des actions?

Les fonctions ont également leurs contraires. Refuser de fournir un renseignement pour se rassurer isole également la personne, ce qui n'empêche pas forcément de fabriquer: l'individu isolé peut être un expert d'autant plus performant qu'il

4. Critique souvent faite au fonctionnalisme.

5. Notamment Conein et Jacopin (1994), Suchman (1987, 1990), Hutchins (1994) et Norman (1993) pour l'action située et Weick et Robert (1993) pour l'interactionnisme.

6. «Fabriquer » est un mot générique qui représente une action directement liée au métier de l'entreprise ; il peut signifier «vendre » dans le cas d'une entreprise de négoce.

Revue internationale P.M.E., vol. 15, ns 3-4, 2002 
travaille seul. Partager les informations pour enrichir la vie sociale ou produire une meilleure qualité peut perturber la marche des services : il y a plus de temps consacré à l'échange, les risques de conflits augmentent. Dévoiler trop tôt un savoirfaire peut gêner son développement et détruire les promesses d'un nouveau projet. Et ainsi de suite.

Dans Vacher (1997, 2000), il a déjà été montré que se positionner à gauche ou à droite du tableau dépend du poids relatif de la culture occidentale ou de l'urgence du contexte ${ }^{7}$ alors que se trouver en haut ou en bas de la grille dépend de l'état d'esprit dans lequel on (le collectif) se trouve: dans le visible (les conséquences prévues d'une pratique) ou dans le ressenti (les conséquences non forcément souhaitées $)^{8}$. Mais ce qui relie ou non les quatre fonctions est essentiel. Nous formulons ainsi l'hypothèse suivante :

Hypothèse: Si les quatre fonctions sont équilibrées, l'information, sa gestion et sa technologie (ici les TIC) auront une place cohérente avec la stratégie et l'organisation de l'entreprise.

\section{Méthodologie}

L'étude globale dans laquelle s'insère ce travail a été menée par une équipe pluridisciplinaire (gestion, ethnographie des organisations, sciences de l'information et de la communication $)^{9}$. Nous avons choisi une position de recherche dite inductive, privilégiant la description et la compréhension de phénomènes à l'aide d'études de cas (Hlady-Rispal, 2000) : nous partons des faits pour faire émerger des tendances sur les usages des TIC.

\subsection{Constitution de l'échantillon}

De décembre 1999 à décembre 2000, nous avons rencontré 42 personnes dans $21 \mathrm{PME}^{10}$. Notre échantillon d'entreprises répond à des exigences de représentativité de l'objet de la recherche selon des critères qui sont plus théoriques que statistiques.

7. Voir notamment d'Iribarne (1989), Jullien (1996), Riveline (1986), Vernant (1996), March (1991) et Saul (1993).

8. Voir Merton (1965).

9. Cf. Boutary et al. (2001, 2002). Les approches développées par le reste de l'équipe sont : les cheminements de la décision d'implantation ou de développement des TIC, l'importance des réseaux et de la proximité géographique pour les PME, les usages commerciaux des TIC, l'analyse critique des sites Web des firmes et l'évolution des positionnements liés à l'intégration des TIC.

10. Nous présentons les résultats pour 19 d'entre elles, car dans 2 entreprises, nous n'avons pas réussi à dégager de fonction pour les TIC. Notons que les entreprises ont été rendues anonymes par l'utilisation de noms fictifs.

Revue internationale P.M.E., vol. 15, nos 3-4, 2002 
Nous avons tenu compte des recommandations de Hlady-Rispal (2001) qui distingue cinq critères : l'homogénéité des cas au regard de la problématique, la variété des cas, la richesse des données qu'ils sont susceptibles de fournir, les spécificités de l'objet de la recherche et l'adoption d'un certain équilibre dans la diversité des cas.

Les traits communs que nous avons retenus pour rendre notre échantillon homogène par rapport aux spécificités de notre objet de recherche sont les suivants :

1) le degré de maturité des PME par rapport aux TIC (elles ont toutes au moins dépassé la phase de réflexion pour passer à l'utilisation);

2) la petite taille des entreprises (elles sont en moyenne de 30 personnes);

3) la situation géographique (région Midi-Pyrénées).

Par ailleurs, les traits enrichissant les données et assurant un certain équilibre dans la diversité des cas sont les suivants :

1) l'appartenance à des secteurs d'activité différents mais représentatifs de la région (agroalimentaire, habillement-accessoires, industrie-bâtiment, informatique-télécom et médical);

2) le niveau d'intégration des TIC (les PME interrogées n'ont pas toutes le même niveau d'équipement ni les mêmes usages ou la même approche stratégique);

3) l'expérience à l'export différent ;

4) le degré d'autonomie (indépendance ou non de l'entreprise).

\subsection{Recueil des données}

Nous avons rencontré dans chaque entreprise plusieurs personnes ayant des fonctions différentes et pour des entretiens durant environ une heure et demie. Outre les entretiens que nous avons menés, nous avons mobilisé les documents commerciaux, les sites Internet et l'observation des lieux mêmes de l'entreprise. Les interventions ont toujours impliqué deux chercheurs et nous avons toujours enregistré puis retranscrit intégralement les entretiens afin de travailler sur le même matériau. L'ensemble des entretiens a été réalisé à partir de trois questionnaires adaptés aux types d'interlocuteurs ${ }^{11}$ :

11. Nous avons rencontré cinq types d'interlocuteur: 1) patron, directeur ou gérant; 2) comptable ou responsable administratif ; 3 ) informaticien ou responsable de la veille technologique ; 4) secrétaire, apprenti ou assistant ; 5) ingénieur de production ou ingénieur commercial.

Revue internationale P.M.E., vol. 15, nºs 3-4, 2002 
1) la connaissance de l'entreprise (son fonctionnement et les conséquences de l'introduction des TIC);

2) l'équipement en TIC de l'entreprise (qui achète, décide, forme, fait la maintenance); et

3) l'utilisation des TIC (qui utilise, dans quel cadre, avec quelle fréquence, avec qui).

Lors des travaux qui ont permis d'élaborer la grille des fonctions de l'information, la méthodologie utilisée était l'observation participante ${ }^{12}$, ce qui permet de dégager facilement les fonctions latentes de l'information (socialiser et rassurer). Le temps laissé à chacun de nos interlocuteurs et la multiplicité des regards posés sur une même entreprise (y compris nos discussions entre chercheurs) nous ont permis de repérer pour chaque entreprise les fonctions des TIC, même latentes.

\subsection{Analyse des données}

De façon classique en étude qualitative, nous avons construit un premier ensemble de tableaux permettant de comparer les entretiens. Pour chaque entreprise, nous avons indiqué les renseignements sur sa situation (taille, type de structure, CA, ancienneté) et sur son métier (type de produit, de clientèle, fournisseur, concurrent, personnel). Pour chaque interlocuteur (de un à trois par entreprise), nous avons rempli le tableau suivant :

TABLEAU 2

Premier niveau d'analyse du discours des PME au sujet des TIC

\begin{tabular}{|c|c|c|c|c|}
\hline TIC Étapes & Réflexion & $\begin{array}{c}\text { Début } \\
\text { d'utilisation }\end{array}$ & $\begin{array}{l}\text { Utilisation : } \\
\quad+2 \text { ans }\end{array}$ & Prospective \\
\hline \multicolumn{5}{|l|}{$\begin{array}{l}\text { Messagerie } \\
\text { électronique }\end{array}$} \\
\hline \multicolumn{5}{|l|}{$\begin{array}{l}\text { Consultation } \\
\text { sur le Web }\end{array}$} \\
\hline Site Web & & & & \\
\hline Autre & & & & \\
\hline
\end{tabular}

Précision sur les outils de gestion, le système d'information*, la stratégie et tout autre sujet à approfondir.

* Par la suite, on utilisera le sigle OGSI pour « outil de gestion et système d'information ». Pour être plus précis, dans le cas de certaines PME, nous aurions dû parler de dispositif informationnel (Roux, 2001).

12. Il s'agit non seulement de passer du temps à observer les acteurs, mais également de participer autant que faire se peut à des projets dans l'entreprise. Cette méthodologie limite le nombre d'entreprises à étudier et demande beaucoup de temps, mais augmente la richesse des résultats (Girin, 1981; Berry, 2000).

Revue internationale P.M.E., vol. 15, nos 3-4, 2002 
Selon l'étape ou le degré de maturité de l'entreprise par rapport aux TIC ${ }^{13}$, les cases comprennent des réponses à la question: "À quoi sert la messagerie électronique, le Web ou autres TIC ? $^{14}{ } \gg$. Le tableau comprend également des précisions sur tout sujet important pour notre interlocuteur. Au fur et à mesure de ce premier travail d'analyse, nous avons repéré des sujets récurrents : la fierté avec laquelle nos interlocuteurs parlent de leur métier et des orientations générales de leur entreprise ainsi que le poids accordé à d'autres outils de gestion et systèmes d'information que les TIC (normes qualité, contrats, règles spécifiques, tableaux de bord, GPAO ${ }^{15}$, etc.). Certaines personnes insistent en effet sur l'importance de ces outils comme facteur de changement de comportement; "L'ISO a été plus fort que l'Internet pour changer les pratiques de communication» résume bien ce point de vue que nous avons souhaité approfondir. Nous avons également dégagé l'importance accordée au support papier. Ce dernier comprend en effet plusieurs actions de gestion décrites spontanément (archivage, support de communication interne ou avec les clients et les fournisseurs, réception et suivi des commandes, etc.).

Nous avons donc repris les entretiens pour les classer dans un nouveau tableau (voir l'annexe I) :

TABLEAU 3

Fonctions assignées aux TIC en lien avec l'organisation et la stratégie

\begin{tabular}{|c|c|c|c|c|c|}
\hline \multicolumn{3}{|c|}{ Situation : } & \multicolumn{3}{|c|}{ Discours sur les : } \\
\hline & de l'entreprise & des TIC & $\begin{array}{l}\text { fonctions } \\
\text { des TIC }\end{array}$ & OGSI & points précis \\
\hline $\begin{array}{l}\frac{8}{\infty} \\
\frac{0}{0} \\
\frac{1}{3}\end{array}$ & $\begin{array}{l}\text { 1) taille } \\
\text { 2) ancienneté } \\
\text { 3) secteur } \\
\text { d'activité }\end{array}$ & $\begin{array}{l}\text { 4) messagerie } \\
\text { électronique, } \\
\text { Web } \\
\text { consultation } \\
\text { 5) site } \\
\text { 6) autre }\end{array}$ & $\begin{aligned} \text { 7) } & \text { fabriquer } \\
8) & \text { montrer } \\
\text { 9) } & \text { socialiser } \\
\text { 10) } & \text { rassurer }\end{aligned}$ & $\begin{array}{l}\text { 11) GPAO } \\
\text { 12) assurance- } \\
\text { qualité } \\
\text { 13) papier }\end{array}$ & $\begin{array}{l}\text { 14) aide financière } \\
\text { 15) orientation générale } \\
\text { de l'entreprise } \\
\text { 16) spécialiste TIC? }\end{array}$ \\
\hline
\end{tabular}

13. Une PME peut se positionner sur plusieurs étapes (p. ex., elle utilise les TIC depuis deux ans, mais précise ce qu'il en était en phase de réflexion et ce qui est prévu pour les années à venir).

14. Nous utilisons la typologie la plus simple : messagerie électronique, Web (consultation et/ou site), autres (cédérom, photos numériques, visioconférence, etc.).

15. Gestion de production assistée par ordinateur. Même s'il s'agit d'un outil de gestion intégré (relié à la gestion commerciale et à la comptabilité), les PMI continuent à l'appeler GPAO et non ERP (Enterprise Resource Planning) ou PGI (Progiciel de gestion intégrée) comme c'est souvent le cas pour les plus grosses entreprises.

Revue internationale P.M.E., vol. 15, ns 3-4, 2002 
Ce nouveau tableau permet de comparer les entreprises sur les critères objectifs de structure (situation de l'entreprise, critères de 1 à 3 ) et d'équipement (situation des TIC, critères 5 et 6 ) ainsi que sur les critères subjectifs de typologie d'usage (discours sur les fonctions des TIC, critères 7 à 10), d'aspects organisationnels, stratégiques et environnementaux (discours sur les OGSI et les points précis, critères 11 à 16). Un dernier critère (typologie) combine les discours sur les orientations (métiers, stratégie, vision) et les éléments objectifs de structures hiérarchiques et de type de relation avec les partenaires selon Ouellet (2001) reprenant les spécificités des PME:

1) les PME entrepreneuriales ayant un marché plutôt local avec un système de direction simple et peu de capacité d'évolution. Ces PME ont des préoccupations opérationnelles et se différencient principalement par leur flexibilité;

2) les PME managériales qui ont des pratiques de gestion traditionnelles et un marché plutôt local avec des exportations simples. Elles évoluent par la production mais ont des difficultés à intégrer les autres innovations ;

3) les PME globales qui naviguent dans un marché international et concurrentiel. Elles mobilisent le réseau de compétences internes et externes pour les décisions stratégiques concernant autant l'orientation de l'entreprise (aspects techniques, commerciaux, organisationnels) que la gestion de l'information.

En combinant les deux tableaux, nous avons à la fois du détail sur les discours, ce qui permet une analyse de contenu et une présentation synthétique des critères permettant de comparer les PME sur le lien entre les TIC, l'organisation et la stratégie (Abell, 1980; Lawrence et Lorsch, 1973; Miller, 1987; Porter, 1999, 2001; Mintzberg, Ahlstrand et Lampel, 1999; Parnel, Wright et Tu, 1996; Strategor, 1997 ; Bergeron, Raymond et Rivard, 2001 ; Jacob, 1999; Venkatraman, 1995 ; Lorino et Tarondeau, 1998). En effet, les discours sur les fonctions des TIC donnent d'importantes indications sur les dimensions sociales et commerciales de l'entreprise (qui communique avec qui, par quel media, pour quelle raison, à quelle fréquence, etc.) ainsi que sur l'importance accordée aux TIC dans les orientations générales (proactivité ou non du dirigeant et de son entourage, usages plus ou moins intégrés aux différentes facettes du métier, etc.). Les compléments sur les OGSI et les points précis permettent de situer les TIC par rapport au reste des préoccupations de l'entreprise. Les critères objectifs donnent les éléments de comparaison classiques complétés par la typologie de Ouellet (2001) pour les spécificités de PME.

Revue internationale P.M.E., vol. 15, nos 3-4, 2002 


\section{Résultats}

\subsection{Présentation des cas}

Nous avons classé les PME selon les fonctions assignées aux TIC par nos interlocuteurs $^{16}: 1$ ) celles qui n'en présentent qu'une, 2) celles qui ont la combinaison de fonctions « fabriquer » et « socialiser», 3) la combinaison «fabriquer » et « montrer», 4) la combinaison « fabriquer » et « rassurer»,5) celles à qui il manque la fonction « rassurer» et 6) celles qui exploitent les quatre fonctions.

Pour chaque entreprise, nous résumons les points clés nous ayant permis de repérer telle ou telle fonction.

1) Les cinq entreprises suivantes ne présentent qu'une seule fonction :

- Vétimin, où «montrer» est la seule fonction dégagée. Un site commercial est en place depuis deux ans ; il est très connu en France, mais il permet de faire un CA inférieur à $5 \%$ du CA total. Le discours, très bien établi, n'est pas encore concrétisé ;

- Médoux, pour la fonction « rassurer ». La messagerie électronique est utilisée depuis trois ans pour garder une trace des dossiers clients et le site reste très peu connu des professionnels, car «c'est tellement cher d'être référencé »;

- Rénaldia, pour « rassurer » (et « détruire ») : «Internet, c'est pour éviter la circulation de papier, je veux tout voir », nous dit le patron même si l'informaticien nous explique que le site est figé depuis trois ans, car Internet est jugé comme un gadget;

- Kadal, pour «fabriquer» (et «isoler»). La messagerie électronique est utilisée de façon intensive avec les fournisseurs, mais le secrétariat se plaint de voir la comptabilité prioritaire pour l'informaticien et ce dernier ne voit dans la messagerie électronique que l'occasion de limiter les coûts et le papier;

- Terrinos, pour « fabriquer » (et « isoler »). Elle comprend l'importance de la logistique à prendre en compte avec les TIC et met en avant l'intérêt de la messagerie électronique pour son faible coût par rapport au papier, mais a «[...] des problèmes de relation avec la société de service informatique, c'est un copain du patron ».

16. Lorsque nous avons repéré les fonctions inverses «détruire» ou «isoler», nous en avons conclu que cela renforçait l'absence des fonctions « fabriquer » ou «socialiser».

Revue internationale P.M.E., vol. 15, n ${ }^{\text {s }} 3-4,2002$ 
2) On observe les deux fonctions «fabriquer » et «socialiser» dans l'entreprise Chouèt : "On a des copains qui nous aident pour l'informatique [...] Internet sert à tout, les enfants jouent avec [...]» Le patron de cette entreprise nous décrivait longuement ses difficultés d'organisation tout en restant pragmatique sur l'usage des TIC: «Le site, c'est une suite logique de notre marketing, il faut être référencé.»

3) Les trois entreprises suivantes exploitent les fonctions «fabriquer» et «montrer»:

- E-c@ve: elle a un site commercial de trois ans d'âge qui permet d'assurer $100 \%$ du CA, mais avec d'importantes pertes dues à une politique de communication très gourmande;

- Akugui : son patron, ancien professeur de marketing, applique les méthodes qu'il enseignait ; la messagerie électronique est en place pour diminuer les coûts, le site n'est qu'une vitrine et la différenciation se fait sur les normes de qualité. Seule la secrétaire arrive à comprendre l'intégration des TIC et des autres techniques dans cet ensemble;

- Batirénov : elle est passée aux TIC pour répondre à une demande pressante des clients. Les archives papier sont conservées à différents endroits et une partie de la gestion est effectuée grâce au Web. On regrette que la messagerie électronique «diminue les contacts» (accentue l'absence de la fonction «socialiser»).

4) Les quatre entreprises suivantes regroupent les fonctions «fabriquer» et «rassurer»:

- Embalto : «Il faudrait déjà penser à envoyer nos tarifs aux VRP (voyageur, représentant, placier)! », nous répond-on quand on pose la question de la messagerie électronique. Par ailleurs, la nécessité d'une base de données en réseau se fait sentir pour avoir « une vraie traçabilité »;

- Marquogam : «Tout doit être noté » grâce à la messagerie électronique, Internet doit servir à faire du marketing classique et à concrétiser tous les efforts faits dans le commercial ;

- Infotek : on y utilise le Web pour «surveiller les concurrents », envoyer des devis et des catalogues, mais un seul poste est utilisé «par peur des virus »;

- Arvin : dans cette entreprise, l'informaticien est très déçu de faire des actualisations du site que personne ne remarque ( « on l'a fait parce qu'il fallait »), mais le responsable administratif précise que la messagerie électronique est utilisée pour communiquer les contrats avec $80 \%$ des clients.

Revue internationale P.M.E., vol. 15, nos 3-4, 2002 
5) Les trois entreprises où manque la fonction « rassurer » sont les suivantes :

- Laserjet : elle utilise la messagerie électronique pour échanger régulièrement des fichiers avec les clients, « on utilise la caméra vidéo et Internet pour faire une plaquette publicitaire en deux heures » et « on s'est formé tout seul »;

- Gépéto : «la messagerie électronique fait partie de la démarche qualité, c'est moins cher et plus rapide », les bases de données sont partagées en interne pour résoudre tout problème client et le Web est utilisé pour faire de la télémaintenance de postes informatiques;

- Copévin : elle a une approche cohérente de la place des TIC par rapport au métier et à son environnement: «Internet, il fallait y aller [...], le Web, c'est moins cher que la pub, on a déjà huit clients par Internet depuis le début de l'année [...], le minitel, c'est quand même plus simple ». L'informatique est présente depuis 1982, le réseau est effectif depuis 1990 et « les prestataires ne viennent plus chez nous, on est sur Internet ou par télétransmission». Le dialogue n'est pas négligé «le code HTML, c'est pas forcément le travail d'une secrétaire mais c'est bien, ça crée des contacts ! ${ }^{17}$.

6) Enfin, les PME où toutes les fonctions sont mises à profit sont les suivantes :

- Coupianum : où les points de vue sur les TIC sont liés aux usages qui peuvent en être faits par rapport au métier de l'entreprise. Selon le patron : «la messagerie électronique permet de faire des miracles» alors que «le Web coûte cher» et que le «site n'est qu'une vitrine». Pour être positionné sur le marché international, il faut pourtant posséder un site vivant, précise le commercial conscient du travail de mise à jour ;

- Cendrillon : où un cadre n'hésite pas à nous parler de problèmes sociaux importants survenus lors de l'implantation de la GPAO. Ces problèmes sont confirmés par le directeur et un autre ingénieur. Cette conscience partagée se retrouve dans la façon dont les TIC sont mobilisées pour chacun : la messagerie électronique pour des échanges de fichiers, le site « 2500 connexions par semaine sur notre site», des envois de photos numériques aux fournisseurs pour faciliter le travail des uns et des autres, etc.;

- Montrinfot : où les procédures sont mises à jour collectivement sur l'intranet, mais « on garde les messageries électroniques pour conserver

17. Chez Copévin, le dirigeant indique qu'il existe un spécialiste TIC, mais la secrétaire précise qu'il s'agit d'elle et qu'elle s'est « formée sur le tas »; elle n'est pas spécialiste. 
une trace, car les clients envoient à n'importe qui en interne». Par ailleurs, nos interlocuteurs font état d'un usage très structuré des TIC : « Pour communiquer avec les clients à l'autre bout du monde », "pour obtenir des affaires » (un site rentabilisé en une vente), etc.

\subsection{Points communs entre les cas et données non significatives}

On retrouve dans les caractéristiques communes des PME interrogées une grande partie des spécificités des PME déjà relevées par Julien (1990), Girard (1997) et Lescure et al., (2001) et des particularités des PME en ce qui concerne les TIC (Bergeron et al., 1998; Ouellet, 2001; Amabile, Gadille et Meissonier, 2000; Blili et Raymond, 1993; BNP Paribas Lease Group, 2002; Gadille et d'Iribane 2000 ; Pouey International, 2000 ; Raymond et Blili, 1992 ; Torrès, 2000 ; Boutary, 2001):

- Elles ont une sophistication structurelle faible avec peu de spécialisation et une centralisation décisionnaire : la direction générale est au maximum tricéphale et lorsque la taille dépasse 10 personnes, elle est aidée par un ou deux collaborateurs, le reste du personnel est opérationnel (administratif ou ouvrier). Elles ont une structure de capital similaire : les dirigeants sont soit créateurs de l'entreprise ou héritiers des créateurs (sauf le cas Kadal). Aucune n'est sous-traitante d'une grande entreprise donneur d'ordres (cette caractéristique est très spécifique à notre échantillon). Elles ont toutes une stratégie plus intuitive qu'explicite (sauf E-c@ve et Akugui), mais se posent de nombreuses questions sur leur métier et leur positionnement par rapport aux concurrents. Elles sont toutes sur des niches avec un savoir-faire clairement identifié;

- Le choix initial des TIC n'est jamais fait pour modifier la stratégie de l'entreprise, mais toujours «pour voir», « sous pression », « pour répondre à un projet précis», «pour des raisons de coût». Le dirigeant a peu de connaissances en TIC et les renseignements de tout ordre ou les questions en cas de difficultés sont toujours demandés en premier lieu à l'entourage proche : le réseau personnel du dirigeant, les partenaires habituels (clients, fournisseurs, voire concurrents) puis les associations d'entreprise et seulement ensuite les pouvoirs publics. Les prestataires de service sont choisis localement. Les sites, lorsqu'ils existent, sont très rarement commerciaux. Les TIC ne remplacent jamais le contact direct avec les clients et les fournisseurs, mais permettent de renforcer la qualité du relationnel.

Une donnée qui n'est pas significative dans notre petit échantillon concerne la demande que font les PME aux aides institutionnelles. On trouve peu de PME ayant reçu des aides et elles sont dans toutes les catégories.

Revue internationale P.M.E., vol. 15, nos 3-4, 2002 


\subsection{Caractéristiques spécifiques selon les catégories}

Nous avons finalement retenu cinq catégories que nous avons nommées d'après les résultats de nos entretiens qui valident l'hypothèse formulée dans la première section: il y a une relation entre le fait de remplir plusieurs fonctions ou non et celui d'avoir des usages des TIC fortement cohérents avec la stratégie et l'organisation de la PME ou non. On peut résumer les catégories dans le tableau suivant:

TABLEAU 4

Catégories de PME selon les fonctions assignées aux TIC

\begin{tabular}{lccccc}
\hline & Borgnes & Hyperactives & Sous-pression & Fébriles & Intégrées \\
\hline Fonction & Une & Fabriquer & Fabriquer & Fabriquer & 3 \\
& seule & + social & + montrer & + rassurer & ou 4 \\
Nombre d'entreprises & 5 & 1 & 3 & 4 & 6 \\
\hline
\end{tabular}

Nous avons classé les PME à qui il manque la fonction «rassurer» dans la même catégorie que celles qui exploitent les quatre fonctions, car nous avons observé dans les deux cas une forte intégration des TIC dans la stratégie et l'organisation de l'entreprise. Deux petites différences sont à noter : la taille et la présence ou non de la GPAO. Les PME n'ayant pas besoin d'être « rassurées » par les TIC (ou de « rassurer » avec les TIC) sont plus petites et n'ont pas de GPAO, elles sont moins confrontées à un besoin de rationaliser leurs pratiques. Elles sont entre les types « entrepreneurial» et «global» alors que les PME ayant les quatre fonctions sont «globales» ou «managériales et globales». Mais toutes les six ont dépassé les deux premières étapes de la reconfiguration de l'organisation grâce aux TIC selon Venkatraman (1995) bien qu'aucune n'ait remis en cause sa stratégie (cinquième et dernière étape). Elles ont toutes un discours sur les orientations générales de l'entreprise, utilisent la messagerie électronique pour intensifier les relations avec les partenaires et leur site Web pour améliorer la prospection (ou la vente). Elles précisent (cinq sur six) que les TIC n'éliminent pas le papier qui a des vertus irremplaçables (consultation - notamment en atelier où les ordinateurs ne sont pas pratiques, archivage, etc.). Quatre sur six sont équipées en TIC depuis plus de deux ans, la même proportion a investi dans les appareils numériques jugés parfaitement complémentaires (photos jointes à la messagerie électronique pour devis, maintenance, catalogue de produits, etc.) et quatre sur six sont certifiées assurance-qualité, certification considérée comme facteur de changement plus important que les TIC. Ces PME «intégrées » ne voient pas l'intérêt d'avoir un spécialiste TIC interne à l'entreprise : elles laissent une personne se former et devenir un amateur chevronné (la secrétaire de Copévin, par exemple) et font appel au réseau (proximité relationnelle et géographique). Elles ne cherchent pas l'autonomie dans ce qui n'est pas directement leur domaine de compétence.

Revue internationale P.M.E., vol. 15, ns 3-4, 2002 
À l'autre extrême, les cinq PME de la première catégorie sont qualifiées de «borgnes», car elles font preuve d'un déséquilibre important entre la stratégie et l'utilisation des TIC. En effet, elles ne voient les TIC que d'un seul œil, c'est-à-dire qu'elles en font une exploitation uniquement locale (que pour la comptabilité ou pour le commercial par exemple). Elles en sont à l'étape initiale d'une éventuelle reconfiguration de l'organisation (Venkatraman, 1995). Trois sur cinq rêvent de pouvoir éliminer le papier grâce aux TIC ${ }^{18}$, elles sont plutôt du secteur de l'habillementaccesssoire (secteur qui a fortement délocalisé ces dernières années) et ont plus de vingt ans (sauf une de cinq ans). Trois sur cinq ont une GPAO qu'elles subissent plus qu'elles ne mobilisent comme moteur organisationnel; Internet est un outil et non pas un élément de la stratégie (Porter, 2001; Venkatraman, 1995 ; Bergeron et al., 1998).

Les trois entreprises « sous pression » de notre échantillon ont en effet choisi les TIC sous contrainte des clients ou des fournisseurs. Elles ont un comportement vif, très réactif, mais elles n'ont pas de projet particulier où les TIC pourraient jouer un rôle comme c'est le cas pour les «intégrées ». Deux sur trois pensent qu'il faut un spécialiste même si elles n'en ont pas. Elles sont plutôt entrepreneuriales et en sont encore à l'exploitation locale des TIC, ce qui est cohérent avec le choix initial des TIC qu'elles n'ont pas encore dépassé.

La catégorie qui semble la plus paradoxale est celle qualifiée de «fébrile», car on observe en effet une grande fébrilité dans les comportements des personnes interrogées et dans leurs discours. Les deux fonctions concernées, «fabriquer» et « rassurer», sont a priori antinomiques : être opérationnel et réactif, ce qui suppose une certaine flexibilité et de la rapidité tout en ayant besoin de certitudes, d'être rassuré (comme utiliser la messagerie électronique pour tracer tous les échanges des collaborateurs avec l'extérieur), ce qui peut prendre un temps considérable. Elles sont toutes managériales et n'abordent aucun problème d'OGSI : elles ne font pas le lien entre leurs nombreux outils de gestion, leur système d'information souvent purement comptable et l'utilisation des TIC. La moitié énonce une stratégie et pense qu'il faut un spécialiste TIC qu'elles n'ont d'ailleurs pas les moyens de se payer.

L'unique PME « hyperactive » est à rapprocher d'un autre cas, Pivert, rencontré dans Vacher (1997), pour les fonctions de l'information et non directement des TIC. Elles ont toutes deux comme point commun d'être dans une logique de l'action comme si opérationnels et dirigeants étaient pris dans un engrenage infernal où personne ne peut s'arrêter de travailler. Les gens travaillent jour et nuit et ont le plus grand mal à se reposer. Cette catégorie est en totale opposition avec celle que l'on pourrait qualifier «d'hyper-rationaliste » et qui correspond à la combinaison des fonctions «montrer» et «rassurer». Cette catégorie n'existe pas dans les cas de PME étudiées ici, mais a déjà été rencontrée dans Vacher (2001) pour les

18. Comme beaucoup croyaient le faire grâce à l'informatique (Vacher, 1997).

Revue internationale P.M.E., vol. 15, nos 3-4, 2002 
fonctions de l'information et de ses systèmes: il s'agissait d'une «bureaucratie mécaniste » (Mintzberg, 1989) dans laquelle l'informatique n'avait aucune portée opérationnelle. On observait en effet une coupure nette entre des opérationnels, gênés par le système dans leurs actions quotidiennes, mais le remplissant consciencieusement de comptes rendus d'action inexploitables, et une direction faisant confiance au système sans comprendre qu'il puisse y avoir des difficultés opérationnelles.

Nous n'avons encore jamais rencontré de cas combinant uniquement les fonctions latentes «socialiser» et « rassurer» ou encore les fonctions «montrer» et «socialiser». Cette absence semble logique ${ }^{19}$ dans la mesure où il serait très paradoxal de mobiliser les TIC à la fois pour afficher une compétence vis-à-vis de l'extérieur et pour avoir un dialogue social (souvent interne) sans aucune fonction opérationnelle directe.

\section{Conclusion}

Les « intégrées » ont une vision à long terme cohérente entre les TIC, l'organisation et la stratégie sans pour autant opérer de réalignement stratégique ${ }^{20}$. Les deux premières étapes de la reconfiguration organisationnelle selon Venkatraman (1995) ont été dépassées : l'exploitation des TIC s'est généralisée à l'ensemble des services de l'entreprise avec une bonne intégration (comme, par exemple, les échanges par messagerie électronique et fichiers attachés entre commerciaux, direction, production et R-D pour améliorer les produits de Gépéto ou la mise à jour du site Web par la secrétaire de Copévin en collaboration avec l'informaticien, le directeur et le marketing). Elles en sont parfois aux étapes trois et quatre: mobilisation des TIC pour revoir leurs réseaux de communication (par exemple, Coupianum où la prospection est faite par Internet, car c'est plus rentable que les salons professionnels, et Laserjet, où photos numériques et fichiers attachés sont utilisés pour le suivi en temps réel des contraintes des clients et des fournisseurs) et amélioration du processus de gestion grâce aux TIC (par exemple, Montrinfot où la mise à jour des procédures d'assurance-qualité est faite par intranet, et Cendrillon, où la préparation des commandes se fait par messagerie électronique et GPAO par les ingénieurs en collaboration avec les magasiniers).

Pour dépasser l'usage statique de notre grille, il est intéressant de se demander dans quelles mesures les autres PME peuvent se rapprocher de cette catégorie «modèle ». Nous avons en effet repris tous les entretiens pour repérer les efforts à

19. Dans une PME, mais peut-être pas dans une administration.

20. Ce qui a déjà été observé par Amabille, Gadille et Meissonier (2000) sur 100 PME en France.

Revue internationale P.M.E., vol. 15, ns 3-4, 2002 
fournir et les compétences à mobiliser, ce que nous détaillons dans notre rapport de recherche (Boutary et al., 2001 et 2002). Ce travail nous a permis de relever quatre processus de passage d'une fonction à l'autre que l'on peut résumer de la façon suivante :

1) concrétiser pour les PME qui sont dans la partie «prouver» de la grille. En effet, si les TIC servent à «montrer » ou à « rassurer» sans lien avec l'action (rentable ou sociale), elles risquent d'être de plus en plus gênantes pour le fonctionnement de l'entreprise ;

2) rationaliser pour les « hyperactives » ou celles qui remplissent une fonction dans la partie « faire » de la grille. En effet, «fabriquer» ou « socialiser» nécessite d'être mis en valeur pour éviter d'être supplanté par la concurrence qui, elle, pourrait faire savoir ce qu'elle ne sait pourtant pas faire ;

3) intérioriser les enjeux de l'entreprise, c'est remplir les fonctions « socialiser» et «rassurer» pour dépasser la seule contrainte de rentabilité et motiver le personnel sur le long terme. Ce processus est valable pour les « sous pression »;

4) rentabiliser pour les entreprises qui mobilisent les TIC uniquement pour « socialiser» ou pour « rassurer ». Cet effort est d'autant plus important que l'entreprise est soumise à de fortes contraintes économiques. Il n'est pas forcément valable pour d'autres formes d'organisation (notamment les associations).

Les «borgnes » et les «fébriles» ont des actions à mener dans toutes les directions. Notre grille de lecture peut alors être mobilisée de façon dynamique.

TABLEAU 5

Processus de passage d'une fonction à l'autre

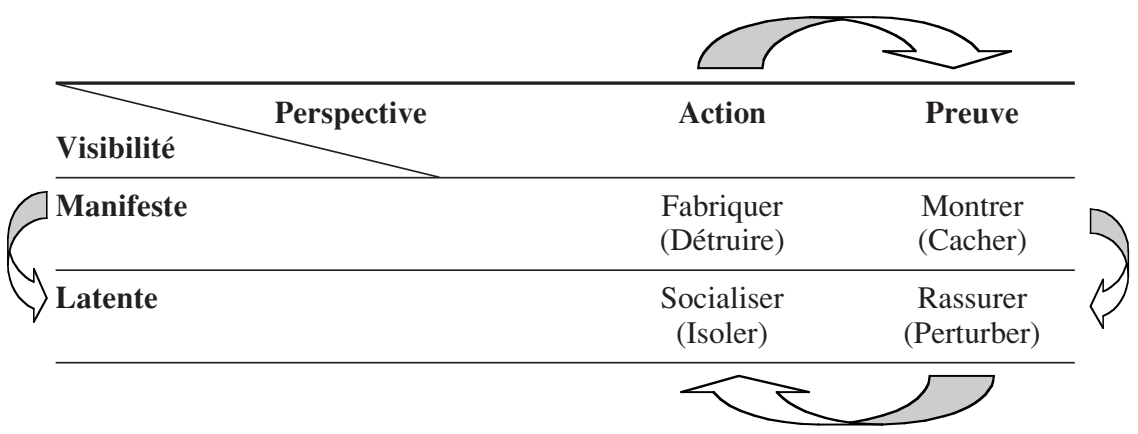

L'approche par les fonctions permet ainsi de montrer le besoin de cohérence stratégique et organisationnelle des TIC en mettant l'accent sur le lien avec les questions que posent les PME sur leur métier, leur positionnement (le processus « rentabiliser »), le lien avec les outils de gestion et les systèmes d'information existants, éléments structurels importants (le processus «rationaliser»), le renforcement du réseau et l'appropriation sociale (les processus « concrétiser» et « intérioriser»). 


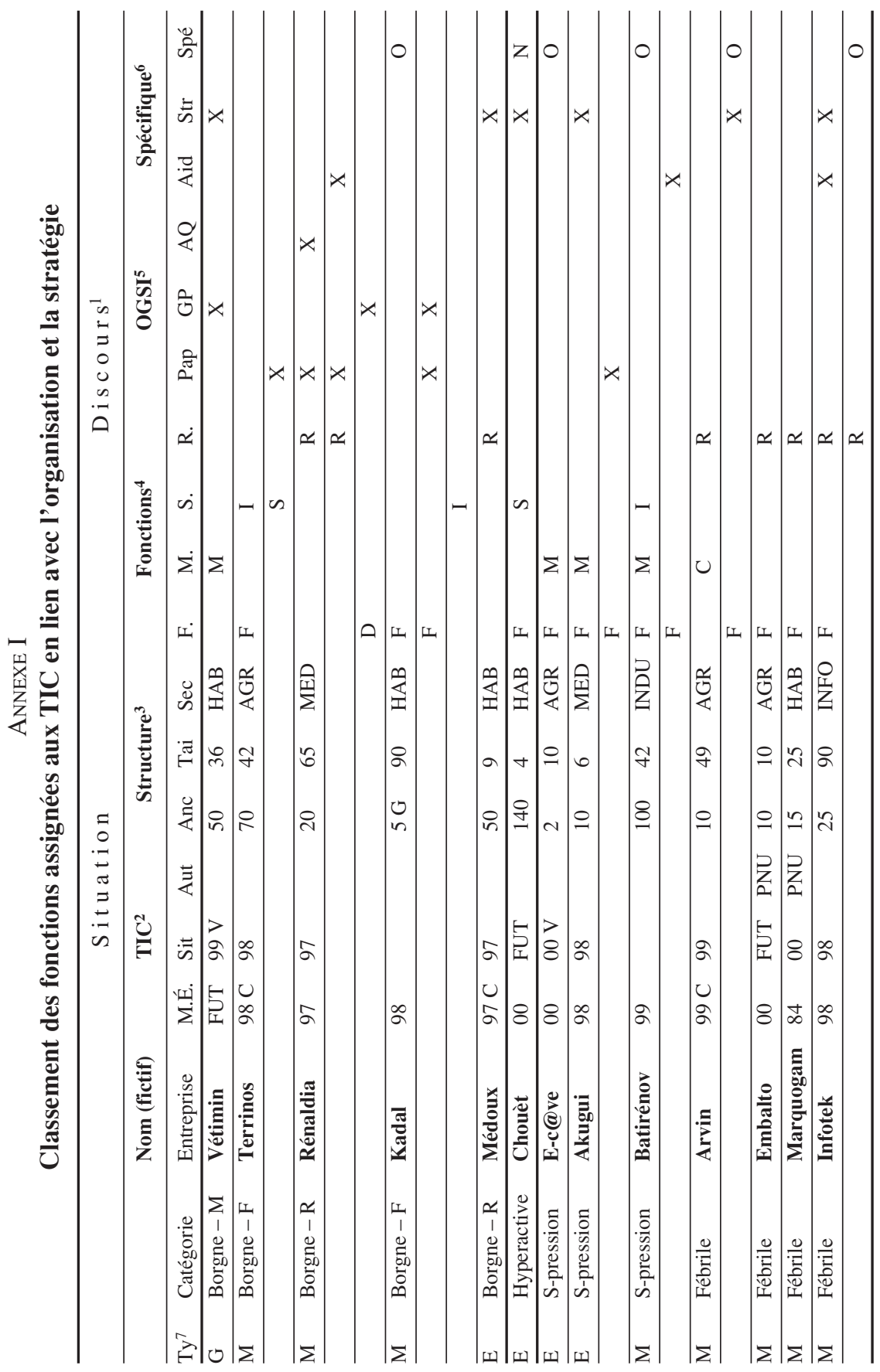

Revue internationale P.M.E., vol. 15, nos 3-4, 2002 


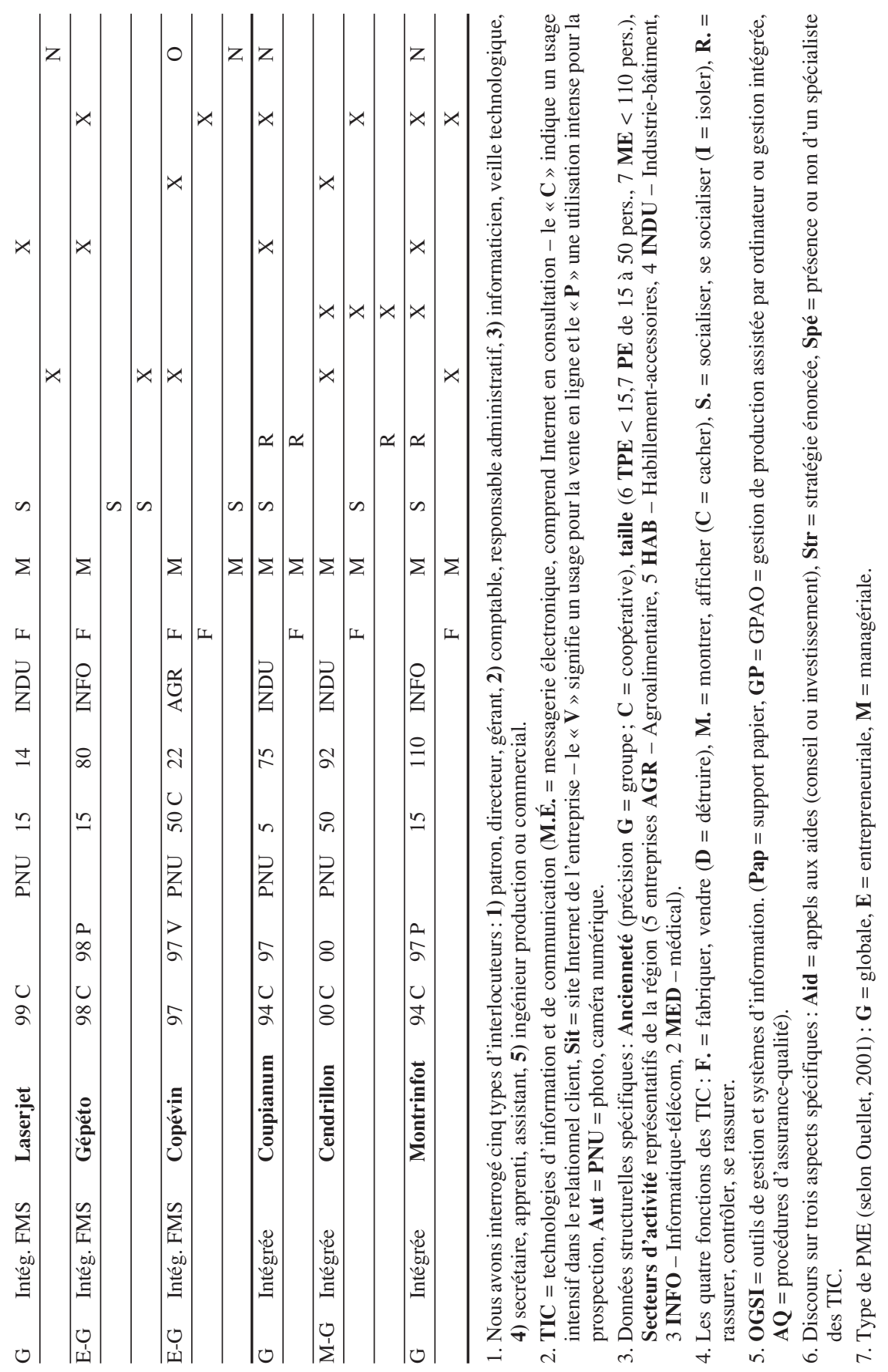

Revue internationale P.M.E., vol. 15, nos 3-4, 2002 


\section{Bibliographie}

Abell, D.F. (1980), Defining the Business: The Starting Point of Strategic Planning, Englewood Cliffs, NJ, Prentice-Hall.

AMABILE, S., M. GADILLE et R. MeISSONIER (2000), «Information, organisation, décision : étude empirique sur les apports des NTIC dans les PME "Internautes" », Systèmes d'information et management, vol. 5, $\mathrm{n}^{\mathrm{o}} 1$, p. 41-59.

AOKI, M. (1988), Information, Incentive and Bargaining Structure in the Japanese Economy, Cambridge, Cambridge University Press,

Bergeron, F., L. RAYMOnd, M. GlAdU et C. LECLERC (1998), «The contribution of information technology to the performance of SMEs : alignment of critical dimensions », Proceedings of the 6th European Conference on Information Systems, Aix-en-Provence, 4 au 6 juin, p. 173-187.

BERGERON, F., L. RAYMOND et S. RIVARD (2001), « Fit in strategic information technology management research : an empirical comparison of perspectives », OMEGA, The International Journal of Management Science, vol. 29, p. 125-142.

BERRY, M. (2000), « Diriger des thèses de terrain », Gérer et comprendre, ${ }^{\circ} 62$, Annales des Mines, décembre, p. 88-97.

BLILI, S. et L. RAYMOND (1993), «Information technology : threats and opportunities for small and medium-sized enterprises », International Journal of Information Management, p. 439-448.

BNP Paribas Lease Group (2002), « Enquête PME-PMI 2001-2002 : nouvelles technologies et Internet », http://www.bnpparibas-leasegroup.com/enquete, janvier, p. 1-2.

BoUTARY, M. (2001), «PME et NTIC : bienfaits et limites d'une gestion de proximité », Actes du $\sigma^{e}$ colloque AIM, Nantes, 7-9 juin, p. 349-355.

BoutARY, M. et al. (2001), Comportement informationnel des PME face aux nouvelles technologies, Rapport de recherche pour la DRIRE Midi-Pyrénées, juin, 189 p.

BoutAry, M. et al. (2002), PME et TIC : des usages aux stratégies, Paris, L'Harmattan, à paraître.

Brousseau, E. et A. RALlET (1997), « Le rôle des technologies de l'information et de la communication dans les changements organisationnels », dans B. Guilhon, P. Huard, M. Orillard et J.-B. Zimmerman (dir.), Économie de la connaissance et organisations - Entreprises, territoires, réseaux, Paris, L'Harmattan, p. 286-309.

CARrière, J.-B. (1990), «La vision stratégique en contexte de PME: cadre théorique et étude empirique », Revue internationale PME, vol. 3, nos 3-4, p. 301-325.

Conein, B. et E. JACOPIN (1994), «Action située et cognition : le savoir en place», Sociologie du travail, $\mathrm{n}^{\circ} 4$, p. 475-500.

D'IRIBARNE, P. (1989), La logique de l'honneur. Gestion des entreprises et traditions nationales, Paris, Le Seuil, 280 p.

FELDMANN, M.S. et J.G. MARCH (1991), «L'information dans les organisations : un signal et un symbole », dans Décisions et organisations, Paris, Les Éditions d'Organisation, p. $255-275$.

Revue internationale P.M.E., vol. 15, ns 3-4, 2002 
GAdille, M. et A. D'IRIBARNE (2000), «La diffusion d'Internet dans les PME. Motifs d'adoption dans les réseaux et ressources mobilisées », Réseaux, $\mathrm{n}^{\circ} 104$, Hermès Science Publication, p. 60-92

GIRARD, B. (1997), « Pourquoi les PME restent-elles PME? », Gérer et comprendre, septembre, p. $33-42$.

GIRIN, J. (1981), «Quel paradigme pour la recherche en gestion? », dans Économies et Sociétés, Tome XII, nos 10-11-12, octobre-novembre, p. 1871-1889.

HLADY-Rispal, M. (2000), «Une stratégie de recherche en gestion: l'étude de cas », Revue française de gestion, janvier-février, p. 61-70.

HLADY-Rispal, M. (2001), Les études de cas: application à la recherche en gestion, Paris, De Boeck, coll. «Méthodes en sciences humaines ».

Hutchins, E. (1994), «Comment le cockpit se souvient de ses vitesses », Sociologie du travail, avril, p. 451-473.

JACOB, R. (1999), « Anatomie d'une rencontre du III' type : gestion stratégique de l'interaction entre les personnes, les NTIC et l'organisation », Actes du colloque sur le changement technologique, Lyon, Entretiens Jacques Cartier, p. 1-22.

JULIEN, P.-A. (1990), « Notes de lecture. Vers une typologie multicritère des PME », Revue internationale PME, vol. 3, nos 3-4, p. 411-425.

JULIEN, P.-A., dir. (1994), PME, bilan et perspectives, Paris, Economica ; Québec, Presses Inter Universitaires.

Julien, P.-A. et M. MARChESNAY (1988), La petite entreprise : principes d'économie et de gestion, Paris, Vuibert.

JULIEN, P.-A., L. RAYMOND, R. JACOB et C. RAMANGALAHY (1997), «Information, stratégies et pratiques de veille technologique dans les PMI », Systèmes d'information et management, vol. 2, $\mathrm{n}^{\mathrm{o}} 2$, p. 63-83.

Jullien, F. (1996), Traité de l' efficacité, Paris, Grasset, 234 p.

LAWRENCE, P.R. et J.W. LORSCH (1973), Adapter les structures de l'organisation, Paris, Les Éditions d'Organisation.

Lescure, M., T. NAKAJima, J. Romano, D. Thierry et H. Zimnovitch (2001), «L'efficacité des PME », Entreprise et Histoire, n 28, Paris, Eska, décembre, p. 89-98.

LORINO, P. et J.-C. TARONDEAU (1998), «De la stratégie aux processus stratégiques », Revue française de gestion, janvier-février, p. 5-17.

MARCH, J.G. (1991), «Système d'information et prise de décision : des liens ambigus », dans Décisions et organisations, Paris, Les Éditions d'Organisation, p. 231-253.

MERTON, R.K. (1965), «L'analyse fonctionnelle en sociologie», dans Éléments de théorie et de méthode sociologique, Paris, Plon, p. 65-139.

MiLLER, D. (1987), « Strategy making and structure : analysis and implications for performance », Academy of Management Journal, vol. 30, n 1, p. 7-32.

MinTZBERG, H. (1989), Le management. Voyage au centre des organisations, Paris, Les Éditions d'Organisation.

Revue internationale P.M.E., vol. 15, nos 3-4, 2002 
MinTZBerg, H., B. AHLSTRAND et J. LAMPEL (1999), Safari en pays stratégie. L'exploration des grands courants de la pensée stratégique, Paris, Village Mondial, 423 p.

MisPelblom-Beyer, F. (1999), Au-delà de la qualité. Démarche qualité, conditions de travail et politiques $d u$ bonheur, $2^{\mathrm{e}}$ édition augmentée, Paris, Syros.

Morgan, G. (1996), Images de l'organisation, Québec, Presses de l'Université de Laval.

NONAKA, I. et H. TAKEUCHI (1995), The Knowledge Creating Company, Oxford, Oxford University Press, 284 p.

NORMAN, D.A. (1993), «Les artefacts cognitifs», dans Les objets dans l'action. De la maison au laboratoire, Textes réunis par B. Conein, N. Dodier et L. Thévenot, Paris, Éditions de l'EHESS, p. 15-34.

Ouellet, P. (2001), «Les affaires électroniques: Tendances et enjeux pour les PME québécoises », Observatoire de Développement économique Canada, décembre, 59 p. (en collaboration avec A. Trudeau).

PARnell, J.A., P. Wright et H.S. Tu (1996), «Beyond the strategy-performance linkage : the impact of the strategy-organization-environment fit on business performance », American Business Review, juin, p. 41-50.

Porter, M. (1999), L'avantage concurrentiel, $2^{\mathrm{e}}$ éd., Paris, Dunod, 642 p.

PORTER, M.E. (2001), «Strategy and the Internet », Harvard Business Review, vol. 79, n 3 , mars, p. 63-78.

Pouey InTERnAtional (2000), «Les PME/PMI », http://www.pouey-international.fr, septembre.

RAYMOND, L. (2000), «Globalisation, économie du savoir et compétitivité : un cadre de veille des tendances et enjeux stratégiques pour la $\mathrm{PME}$ », Revue internationale de gestion, vol. 25, $\mathrm{n}^{\circ} 2$, p. 29-38.

RAYMOND, L. et S. BLILI (1992), «Les systèmes d'information dans les PME: synthèse et apports de la recherche », Revue Organisation, été, p. 146-166.

Riveline, C. (1986), «Les lunettes du prince», Gérer et comprendre, juin, p. 5-15.

RouX, A. (2001), «Systèmes d'information, dispositifs informationnels et formes organisationnelles émergentes : mise en articulation », Actes du Colloque Organisation et communication, Rennes, novembre.

SAUL, J. (1993), Les bâtards de Voltaire. La dictature de la raison en Occident, Paris, Éditions Payot et Rivages, 653 p.

Strategor (1997), Politique générale de l'entreprise, 3e éd., Paris, Dunod, 551 p.

Suchman, L. (1987), Plans and Situated Actions, Cambridge, Cambridge University Press.

SuCHMAN, L. (1990), «Plans d'action. Problèmes de représentation de la pratique en sciences cognitives », Raisons Pratiques 1, Les formes de l'action, p. 149-170.

TORRÈs, O. (2000), «Du rôle et de l'importance de la proximité dans la spécificité de gestion des PME», Colloque international francophone de la PME, Lille, 25-27 octobre.

Revue internationale P.M.E., vol. 15, $\mathrm{n}^{\text {os }} 3-4,2002$ 
VACHER, B. (1997), La gestion de l' information en entreprise. Enquête sur l'oubli, l'étourderie, la ruse et le bricolage organisés, Paris, ADBS Éditions, 231 p.

VACHER, B. (2000), «Utilisation, organisation et malentendus de l'information et de ses systèmes », dans Techniques documentaires, Paris, Weka.

VACHER, B. (2001), «Faire ou prouver? L'écrit de l'assurance qualité », dans Le langage dans les organisations - Une nouvelle donne, textes réunis par S. Pène, A. Borzeix, B. Fraenkel, Paris, L’Harmattan, coll. «Langage et travail», p. 115-130.

VENKATRAMAN, N. (1995), «Reconfigurations d'entreprises provoquées par les technologies de l'information », dans L'entreprise compétitive au futur. Technologies de l' information et transformation de l' organisation, Paris, Les Éditions d'Organisation, p. 151-195.

VERnANT, J.-P. (1996), Entre mythe et politique, Paris, Le Seuil, 635 p.

WEICK, K.E. et K.H. RoBERTS (1993), «Collective mind in organisations: Heedful interrelating on flight decks », Administrative Science Quarterly, vol. 38, $\mathrm{n}^{\circ} 3$, septembre, p. 357-381.

Revue internationale P.M.E., vol. 15, nos 3-4, 2002 